Fizika Nizkikh Temperatur, 1999, v. 25, No 10, p. 1021-1026

\title{
Optical generation of the first and second sounds in superfluid ${ }^{3} \mathrm{He}-{ }^{4} \mathrm{He}$ solutions
}

\author{
T. Kh. Salikhov \\ Department of Physics, Dushanbe State Teacher's Training Institute, \\ 121 Rudaki Str., 734003 Dushanbe, Tadjikistan \\ E-mail: sebak@td.silk.org
}

Received September 15, 1998, revised March 16, 1999

\begin{abstract}
An optical method of generation of the first and second sounds in superfluid ${ }^{3} \mathrm{He}-{ }^{4} \mathrm{He}$ solutions is proposed. The method is based on illumination by amplitude-modulated laser beam with wavelength corresponding to a region of absorption. Numerical calculations of pressure and temperature as a function of time are carried out. It was found that the presence of ${ }^{3} \mathrm{He}$ isotopes in the solution provides strong coupling between these parameters and, as a result, the waves of the first sound involve oscillations of the temperature, while the waves of the second sound involve oscillations of the pressure.
\end{abstract}

PACS: 67.60.-g

\section{Introduction}

As in He-II, the waves of the first and the second sounds can propagate in superfluid ${ }^{3} \mathrm{He}-{ }^{4} \mathrm{He}$ solutions [1]. Rudavsky and Serbin have considered generation of acoustical waves involving oscillations of pressure and temperature in the solutions [2]. They extended the method, which was developed by Lifshitz [3] for superfluid helium, to the superfluid ${ }^{3} \mathrm{He}-{ }^{4} \mathrm{He}$ solutions. It was shown that a surface with periodically varying temperature generates waves of both the first and second sounds in the solutions, and that intensities of the waves are of the same order of magnitude. Such an important difference between the solutions and He-II is due to strong coupling between the density of the medium and the concentration of ${ }^{3} \mathrm{He}$ isotopes. This coupling gives rise to a strong relation between the oscillating parts of the pressure $P^{\prime}(t, \mathbf{r})$ and the temperature $T^{\prime}(t, \mathbf{r})$. As a result, the waves of the second sound lead to oscillations of the pressure, while the waves of the first sound give rise to oscillations of the temperature. This effect has been observed and studied in detail experimentally [4,5]. Because of its simplicity, this method is now widely used.

In this paper we propose an optical method allowing one to generate waves of the first and second sounds in superfluid ${ }^{3} \mathrm{He}-{ }^{4} \mathrm{He}$ solutions. The advantage of the proposed method is that it is contactless. It is worth mentioning that there are many theoretical and experimental studies of optical generation of acoustic waves in classical liquids and solids (see the review article [7]). In the case of He-II such a method of generation of the second sound was studied theoretically [8]. It was shown that waves of the second sound can be generated by laser radiation. In this paper our aim is to extend the results obtained in Ref. 8 to superfluid ${ }^{3} \mathrm{He}-{ }^{4} \mathrm{He}$ solutions.

Let us assume that the system under consideration has regions of absorption in various ranges of the spectrum (for example, the ${ }^{4} \mathrm{He}$ isotope has an absorption line in the ultraviolet region $\sim 600 \AA$ with the absorption coefficient about $1 \mathrm{~cm}^{-1}$ (Ref. 9 and 10) and is described by the absorption coefficient $\alpha$. Then, generation of waves of the first and second sounds by amplitude-modulated laser radiation (for example, an excimer laser) is possible. The illuminated medium due to periodically changing temperature and thermal expansion emits waves of the first and second sounds. Due to the strong coupling between $P^{\prime}(t, \mathbf{r})$ and $T^{\prime}(t, \mathbf{r})$, the oscillations of one of these parameters give rise to the oscillations of the other parameter.

\section{Equations for waves of the first and second sounds}

We assume a solution of ${ }^{3} \mathrm{He}-{ }^{4} \mathrm{He}$ to be confined in a cylindrical container with volume $V$ and radius 
$R$. A monochromatic laser beam with the amplitude and Gaussian shape of the spatial distribution, changing in time is assumed to move along the axis of the cylinder. Absorption in the system provides dissipation of the radiation energy and generation of acoustic waves according to the thermal mechanism described in Ref. 7. We assume that the amplitudes of oscillations of hydrodynamic parameters are much lower than their average magnitudes. This allows us to apply a system of linear hydrodynamic equations. This system consists of the mass conservation law, the equation describing the movement of the liquid, the equation for entropy taking into account the heat source, the equation for concentration taking into account the solvent only to the normal mode, and the equation for the superfluid component [1]. Further, we ignore the dissipation terms in the hydrodynamic equations and use the system of equation [1]

$$
\begin{gathered}
\frac{\partial \rho}{\partial t}+\rho_{s} \nabla \cdot \mathbf{v}_{s}+\rho_{n} \nabla \cdot \mathbf{v}_{n}=0, \\
\rho_{s} \frac{\partial \mathbf{v}_{s}}{\partial t}+\rho_{n} \frac{\partial \mathbf{v}_{n}}{\partial t}+\nabla P^{\prime}=0, \\
\rho_{0} \frac{\partial \sigma^{\prime}}{\partial t}+\sigma_{0} \rho_{s} \nabla \cdot\left(\mathbf{v}_{n}-\mathbf{v}_{s}\right)=\frac{1}{T_{0}} f(t, \mathbf{r}), \\
\sigma_{0} \frac{\partial c^{\prime}}{\partial t}-c_{0} \frac{\partial \sigma^{\prime}}{\partial t}=-\frac{c_{0}}{\rho_{0} T_{0}} f(t, \mathbf{r}), \\
\frac{\partial \mathbf{v}_{s}}{\partial t}+\nabla\left(\mu-\frac{Z c}{\rho}\right)=0,
\end{gathered}
$$

where $\rho=\rho_{s}+\rho_{n}, \sigma_{0}$ and $c_{0}$ are the average values of the mass, the entropy per unit mass, and the concentration, $\rho^{\prime}, \sigma^{\prime}$, and $c^{\prime}$ are their amplitudes, $\rho_{s}$ and $\rho_{n}$ are the densities and $\mathbf{v}_{s}$ and $\mathbf{v}_{n}$ are the velocities of the superfluid and the normal components, respectively, and

$$
Z=\rho\left(\mu_{3}-\mu_{4}\right), \quad \mu=c \mu_{3}+(1-c) \mu_{4},
$$

where $\mu_{3,4}$ is the chemical potential of ${ }^{3} \mathrm{He}$ and ${ }^{4} \mathrm{He}$, and $f(t, \mathbf{r})$ is the heat source due to the absorbed light.

We then ignore the terms on the right side of (4), i.e. we consider the case of small concentration. Excluding from (1)-(5) $\mathbf{v}_{s}, \mathbf{v}_{n}$, and $c^{\prime}$ and using the thermodynamic expression [1] which connects $\mu^{\prime}$ and $\sigma^{\prime}$ with the perturbations of pressure $P^{\prime}$ and temperature $T^{\prime}$, we obtain

$$
\begin{aligned}
& \frac{\partial^{2} P^{\prime}}{\partial t^{2}}-C_{1}^{2}\left(1+\frac{\rho_{s}}{\rho_{n}} \beta^{2}+\frac{\beta \alpha_{T} C_{2}^{2}}{\bar{\sigma}}\right) \Delta P^{\prime}+ \\
& +\frac{\rho_{0} \beta C_{P} C_{1}^{2} C_{2}^{2}}{T_{0} \bar{\sigma}} \Delta T^{\prime}=-\frac{\beta C_{1}^{2}}{T_{0} \sigma_{0}} \frac{\partial f}{\partial t}, \\
& \frac{\partial^{2} T^{\prime}}{\partial t^{2}}-C_{2}^{2}\left(1-\frac{\beta \alpha_{T} C_{2}^{2}}{\bar{\sigma}}\right) \Delta T^{\prime}-\frac{T_{0}}{\rho_{0} C_{P}} \times \\
& \times\left[\left(C_{1}^{2}-C_{2}^{2}\right) \alpha_{T}+\frac{\rho_{s}}{\rho_{n}} \alpha_{T} \beta^{2} C_{1}^{2}-\frac{\rho_{s}}{\rho_{n}} \beta \bar{\sigma}+\frac{\beta \alpha_{T}^{2} C_{1}^{2} C_{2}^{2}}{\bar{\sigma}}\right] \Delta P^{\prime}= \\
& =\frac{1}{\rho_{0} \sigma_{0} C_{P}}\left(\bar{\sigma}-\beta \alpha_{T} C_{1}^{2}\right) \frac{\partial f}{\partial t}, \\
& \bar{\sigma}=\sigma_{0}-c_{0} \frac{\partial \sigma}{\partial c}, \beta=\frac{\bar{\sigma}}{\rho_{0}}\left(\frac{\partial \rho}{\partial T}\right)_{P_{C}}\left(\frac{\partial T}{\partial \sigma}\right)_{P_{C}}+\frac{c_{0}}{\rho_{0}}\left(\frac{\partial \rho}{\partial c}\right)_{P T} .
\end{aligned}
$$

Heat sources of sound are taken into account on the right-hand sides of Eqs. (7) and (8). The system of wave equations (7) and (8) describes the dependence of temperature and pressure of superfluid ${ }^{3} \mathrm{He}-{ }^{4} \mathrm{He}$ solution on time and spatial coordinates when an electromagnetic wave with varying amplitude and wavelength corresponding to the absorption range propagates in the sample. For example, the terms with the factors $\Delta T^{\prime}$ in Eq. (7) and $\Delta P^{\prime}$ in Eq. (8) due to large value of $|\beta| \approx 0.1-0.4$ provide the strong relation between the amplitudes of temperature and pressure as the first and second sound waves propagate.

\section{Solution of the system of acoustical equations for the first and second sounds. Analysis and numerical results}

The heat sources in Eqs. (7) and (8) provide a transfer of electromagnetic wave energy to acoustical energy. The transfer goes according to the heat mechanism, i.e., due to the heat expansion, the strong dependence of the density on the concentration $\left(\left|\left(c_{0} / \rho_{0}\right)(\partial \rho / \partial c)\right|>>\alpha_{T}\right)$, and the periodical changing of the temperature at the surface of the illuminated volume.

Further, for simplicity we ignore the variation of the intensity in the medium caused by absorption. We assume that the laser beam propagates along the axis of the cylindrical container. This allows us to set 


$$
f(t, \mathbf{r})=\alpha I_{0} \varphi(t) \varphi_{1}(r)
$$

where $\varphi(t)$ is a function describing the oscillating part of the intensity, $\varphi_{1}(\mathbf{r})=\left(2 / \pi w^{2}\right) \exp \left[-2 r^{2} / w^{2}\right]$ describes its radial distribution, and $I_{0}$ and $w$ are the power and radius of the stretching of the light beam, respectively. We also assume that w is much smaller than the radius of the cylindrical container.

Taking the Hankel transfer of Eqs. (7) and (8) with respect to $r$

$$
\bar{A}^{\prime}(t, s)=\int_{0}^{\infty} \bar{A}^{\prime}(t, r) J_{0}(r s) r d r
$$

where $J_{0}$ is the Bessel function, and $\bar{A}^{\prime}(t, s)=\overline{T^{\prime}}(t, s)$ or $\bar{P}^{\prime}(t, s)$, we obtain

$$
\begin{gathered}
\frac{\partial^{2} \bar{P}^{\prime}}{\partial t^{2}}+C_{1}^{2} s^{2}\left(1+\frac{\rho_{s}}{\rho_{n}} \beta^{2}+\frac{\alpha_{T} \beta C_{2}^{2}}{\bar{\sigma}}\right) \bar{P}^{\prime}- \\
-\frac{\rho_{0} \beta C_{P} C_{2}^{2} C_{1}^{2} s^{2}}{T_{0} \bar{\sigma}} \bar{T}^{\prime}=-\frac{\alpha I_{0} \beta C_{1}^{2} \varphi_{1}}{T_{0} \sigma_{0}} \frac{\partial \varphi}{\partial t}, \quad \text { (12) } \\
\frac{\partial^{2} \bar{T}^{\prime}}{\partial t^{2}}+C_{2}^{2} s^{2}\left(1-\frac{\alpha_{T} \beta C_{1}^{2}}{\bar{\sigma}}\right) \bar{T}^{\prime}+\frac{T_{0} s^{2}}{\rho_{0} C_{P}}\left[\alpha_{T}\left(C_{1}^{2}-C_{2}^{2}\right)+\right. \\
\left.+\frac{\rho_{s}}{\rho_{n}} \alpha_{T} \beta^{2} C_{1}^{2}-\frac{\rho_{s}}{\rho_{n}} \beta \bar{\sigma}+\frac{\beta \alpha_{T}^{2} C_{1}^{2} C_{2}^{2}}{\bar{\sigma}}\right] \bar{P}^{\prime}= \\
=\frac{\alpha I_{0} \varphi_{1}(s)}{\rho_{0} \sigma_{0} C_{P}}\left(\bar{\sigma}-\beta \alpha_{T} C_{1}^{2}\right) \frac{\partial \varphi}{\partial t} .
\end{gathered}
$$

It is assumed that $T^{\prime}(r) \rightarrow 0$ and $\partial T^{\prime}(r) / \partial r \rightarrow 0$ as $r \rightarrow \infty$. We also use the notation

$$
\varphi_{1}(s)=\int_{0}^{\infty} \varphi_{1}(r) J_{0}(r s) r d r=\frac{1}{2 \pi} \exp \left(-w^{2} s^{2} / 8\right) .
$$

We will consider two cases: instantaneous switching and harmonical law.

\section{Instant switching}

In the case of instantaneous switching $\varphi(t)$ is equal to the Heaviside unit step function $\theta(t)$ and its time derivative is equal to the Dirac function $\delta(t)$. Using the Laplace transform of Eqs. (12) and (13)

$$
\hat{A}(p, s)=\int_{0}^{\infty} d t \exp (-p t) \bar{A}^{\prime}(t, s)
$$

with the initial conditions $T^{\prime}(0, s)=P^{\prime}(0, s)=0$, we obtain

$$
\begin{gathered}
A_{11} \hat{P}(p, s)+A_{12} \hat{T}(p, s)=-\frac{\alpha I_{0} \beta C_{1}^{2} \varphi_{1}(s)}{T_{0} \sigma_{0}}, \\
A_{21} \hat{P}(p, s)+A_{22} \hat{T}(p, s)=\frac{\alpha I_{0} \varphi_{1}(s)}{\rho_{0} \sigma_{0} C_{P}}\left(\bar{\sigma}-\beta \alpha_{T} C_{1}^{2}\right),
\end{gathered}
$$

where the coefficients $A_{i j}$ are defined as

$$
\begin{aligned}
& A_{11}=p^{2}+s^{2} C_{1}^{2}\left(1+\frac{\rho_{s}}{\rho_{n}} \beta^{2}+\frac{\alpha_{T} \beta C_{2}^{2}}{\bar{\sigma}}\right), \\
& A_{12}=-\frac{\rho_{0} \beta C_{P}}{T_{0} \bar{\sigma}} C_{1}^{2} C_{2}^{2} s^{2}, \\
& A_{21}=\frac{T_{0} s^{2}}{\rho_{0} C_{P}} \times \\
& \times\left[\alpha_{T}\left(C_{1}^{2}-C_{2}^{2}\right)+\frac{\rho_{s}}{\rho_{n}} \alpha_{T} \beta^{2} C_{1}^{2}-\frac{\rho_{s}}{\rho_{n}} \beta \bar{\sigma}+\frac{\beta \alpha_{T}^{2} C_{1}^{2} C_{2}^{2}}{\bar{\sigma}}\right], \\
& A_{22}=p^{2}+s^{2} C_{2}^{2}\left(1-\frac{\alpha_{T} \beta C_{1}^{2}}{\bar{\sigma}}\right) .
\end{aligned}
$$

The system of linear equations (16) has a solution if $A_{11} A_{22}-A_{12} A_{21}=0$. This condition can be rewritten as

$$
p^{4}+p^{2} s^{2}\left(C_{1}^{2}+C_{2}^{2}+\frac{\rho_{s}}{\rho_{n}} \beta^{2} C_{1}^{2}\right)+s^{4} C_{1}^{2} C_{2}^{2}=0 .
$$

The roots of Eq. (17) can be found as $p_{1,2}^{2}=-s^{2} U_{1,2}^{2}$

$$
\begin{gathered}
U_{1}^{2}=C_{1}^{2}\left(1+\frac{C_{1}^{2}}{C_{1}^{2}-C_{2}^{2}} \frac{\rho_{s}}{\rho_{n}} \beta^{2}\right), \\
U_{2}^{2}=C_{2}^{2}\left(1+\frac{C_{1}^{2}}{C_{1}^{2}-C_{2}^{2}} \frac{\rho_{s}}{\rho_{n}} \beta^{2}\right)^{-1},
\end{gathered}
$$


where $U_{1,2}$ are the velocities of the first and second sounds, respectively. It should be mentioned that we have simplified expressions for $p_{1,2}^{2}$ using the small parameter $\left(\rho_{s} / \rho_{n}\right) \beta^{2}<<1$. A solution of Eq. (16) is given by

$$
\begin{gathered}
\hat{P}(p, s)=-\frac{\alpha I_{0} \beta C_{1}^{2}}{T_{0} \sigma_{0}} \frac{\varphi_{1}(s) p^{2}}{\left(p^{2}-p_{1}^{2}\right)\left(p^{2}-p_{2}^{2}\right)}, \\
\hat{T}(p, s)=\frac{\alpha I_{0} \varphi_{1}(s)}{\rho_{0} \sigma_{0} C_{P}} \frac{p^{2}\left(\bar{\sigma}-\alpha_{T} \beta C_{1}^{2}\right)+\bar{\sigma} s^{2} C_{1}^{2}}{\left(p^{2}-p_{1}^{2}\right)\left(p^{2}-p_{2}^{2}\right)} .
\end{gathered}
$$

To find $P^{\prime}(t, r)$ and $T^{\prime}(t, r)$ one should use the inverse Laplace and Hankel transforms of Eqs. (20) and (21). The inverse Laplace transform gives rise to

$$
\begin{aligned}
& \bar{P}^{\prime}(t, s)=-\frac{\alpha I_{0} \beta C_{1}^{2} \varphi_{1}(s)}{T_{0} \sigma_{0}} \frac{1}{2 \pi i} \int_{\sigma-i \infty}^{\sigma+i \infty} \frac{d p p^{2} \exp (p t)}{\left(p^{2}-p_{1}^{2}\right)\left(p^{2}-p_{2}^{2}\right)}= \\
& =-\frac{\alpha I_{0} \beta C_{1}^{2} U_{1}}{T_{0} \sigma_{0}\left(U_{1}^{2}-U_{2}^{2}\right)}\left[\sin \left(s U_{1} t\right)-\frac{U_{2}}{U_{1}} \sin \left(s U_{2} t\right)\right] \frac{\varphi_{1}(s)}{s}, \\
& \bar{T}^{\prime}(t, s)=\frac{\alpha I_{0} \bar{\sigma}}{\rho_{0} \sigma_{0} C_{P} U_{2}} \times \\
& \times\left[\left(1+G_{2}\right) \sin \left(s U_{2} t\right)-G_{1} \frac{U_{2}}{U_{1}} \sin \left(s U_{1} t\right)\right] \frac{\varphi_{1}(s)}{s},
\end{aligned}
$$

where

$$
\begin{gathered}
G_{1}=\frac{C_{1}^{2}\left(\alpha_{T} \beta U_{1}^{2}-\overline{\sigma \gamma}\right)}{\bar{\sigma}\left(U_{1}^{2}-U_{2}^{2}\right)}, \quad G_{2}=\frac{C_{1}^{2}\left(\alpha_{T} \beta U_{2}^{2}-\overline{\sigma \gamma}\right)}{\bar{\sigma}\left(U_{1}^{2}-U_{2}^{2}\right)}, \\
\bar{\gamma}=\frac{C_{1}^{2}}{C_{1}^{2}-C_{2}^{2}} \frac{\rho_{s}}{\rho_{n}} \beta^{2}=\frac{U_{1}^{2}-C_{1}^{2}}{C_{1}^{2}}=\frac{C_{2}^{2}-U_{2}^{2}}{U_{2}^{2}}>0 .
\end{gathered}
$$

Using the Hankel transfer of Eqs. (22) and (23), we can write

$$
P(t, r)=-\frac{\alpha I_{0} \beta C_{1}^{2} U_{1}}{T_{0} \sigma_{0}\left(U_{1}^{2}-U_{2}^{2}\right)} \times
$$

$$
\times \int_{0}^{\infty} d s J_{0}(s r) \varphi_{1}(s)\left[\sin \left(s U_{1} t\right)-\frac{U_{2}}{U_{1}} \sin \left(s U_{2} t\right)\right]
$$

$$
T^{\prime}(t, r)=\frac{\alpha I_{0} \bar{\sigma}}{\rho_{0} C_{P} \sigma_{0} U_{2}} \times
$$

$$
\times \int_{0}^{\infty} d s J_{0}(s r) \varphi_{1}(s)\left[\left(1+G_{2}\right) \sin \left(s U_{2} t\right)-G_{1} \frac{U_{2}}{U_{1}} \sin \left(s U_{1} t\right)\right] .
$$

If we set in Eq. (24) $U_{1}^{2} \approx C_{1}^{2}$, we obtain

$$
\bar{\gamma}=0, G_{2}=\frac{\alpha_{T} \beta U_{1}^{2} U_{2}^{2}}{\bar{\sigma}\left(U_{1}^{2}-U_{2}^{2}\right)}=G, \quad G_{1}=G \frac{U_{1}^{2}}{U_{2}^{2}},
$$

and Eq. (26) can be written as

$$
T^{\prime}(t, r)=\frac{\alpha I_{0} \bar{\sigma}}{\rho_{0} C_{P} \sigma_{0} U_{2}} \times
$$

$\times \int_{0}^{\infty} d s J_{0}(s r) \varphi_{1}(s)\left[(1+G) \sin \left(s U_{2} t\right)-G \frac{U_{1}}{U_{2}} \sin \left(s U_{1} t\right)\right]$.

The first term in Eq. (25) corresponds to impulse of the first sound, and the second term corresponds to the variation in temperature of the first sound wave. It always has an opposite phase. Since $\beta \approx\left(c_{0} / \rho_{0}\right)(\partial \rho / \partial c)<0$, the amplitude of the first sound, $P_{\max }=-\alpha I_{0} \beta U_{1}^{3} / T_{0} \sigma_{0}\left(U_{1}^{2}-U_{2}^{2}\right)$, is always positive. The first term in the expression for $T^{\prime}(t, r)$ is a heat impulse of the second sound, while the second term corresponds to the variation of pressure when temperature waves pass in the considered solution. It is worth mentioning that according to Eq. (26) or Eq. (28), a sign of the phase of $P_{(2)}^{\prime}$ depends of the sign of $G_{1}$ and can either be opposite to the sign of the phase of the impulse or coincide with it. In the range of temperature and concentration where $\alpha_{T}<0$ the phases of $P_{(2)}^{\prime}(t, r)$ and $T_{(2)}^{\prime}(t, r)$ are opposite to each other, but for $\alpha_{T}>0$, which provides $\alpha_{T} \beta U_{1}^{2}<\overline{\sigma \gamma}$ or $G_{1}<0$, the phases coincide.

Figures $1, a, b$ show the amplitudes of the pressure and temperature against time. The calculation was carried out on the basis of Eqs. (25) and (28) for $r=1 \mathrm{~cm}, w=0.05 \mathrm{~cm}, \alpha=1 \mathrm{~cm}^{-1}, I_{0}=1 \mathrm{~W}$, $T_{0}=1.5 \mathrm{~K}, \alpha_{T}=-12 \cdot 10^{-3} \mathrm{~K}^{-1}, \beta=-0.3$ (Ref. 11), 

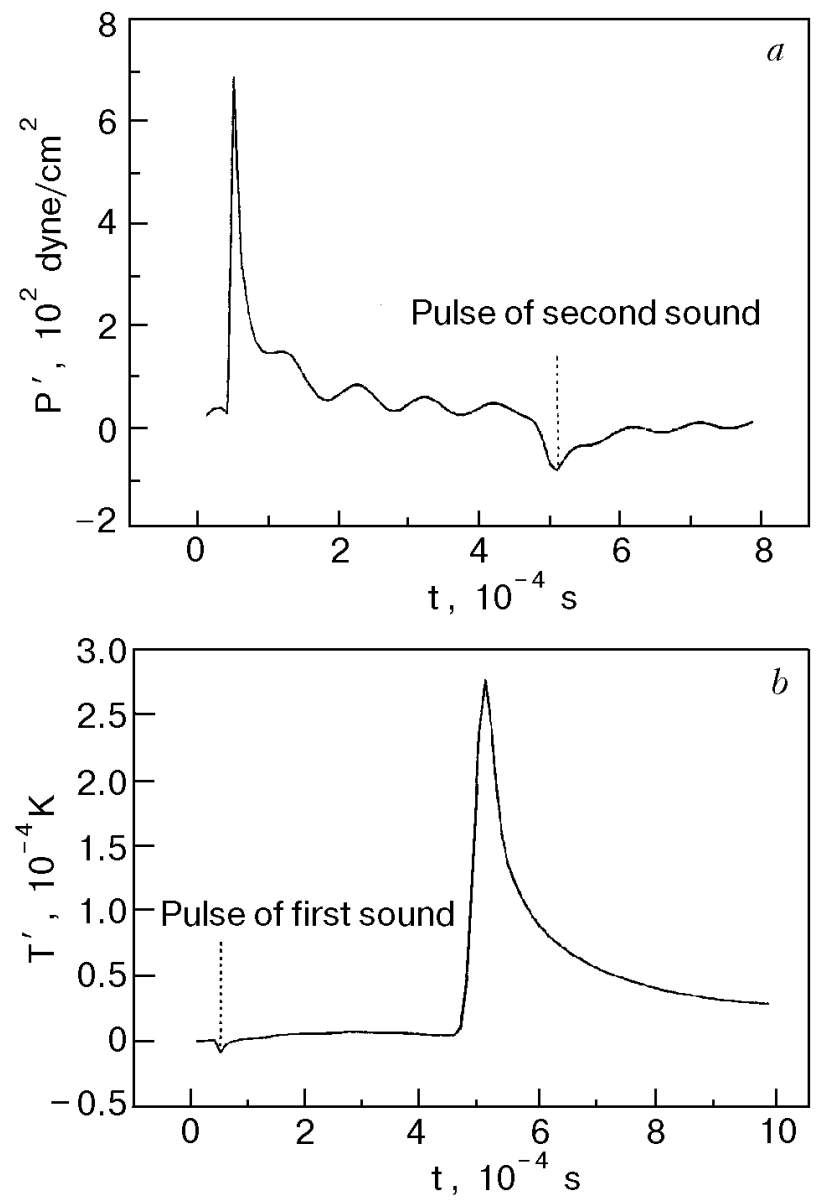

Fig. 1. Pressure (a) and temperature (b) against time for a superfluid ${ }^{3} \mathrm{He}-{ }^{4} \mathrm{He}$ solution. The calculation was carried out for instantaneous switching and $r=1 \mathrm{~cm}$.

$U_{1}=220 \mathrm{~m} / \mathrm{s}, \quad U_{2}=20 \mathrm{~m} / \mathrm{s}, \quad \bar{\sigma}=620 \mathrm{~J} /(\mathrm{kg} \cdot \mathrm{K})$ (Ref. 6) $, \sigma_{0}=2.3 \cdot 10^{3} \mathrm{~J} /(\mathrm{kg} \cdot \mathrm{K}), \rho_{0}=0.12 \mathrm{~g} / \mathrm{cm}^{3}$, and $C_{p}=3 \cdot 10^{3} \mathrm{~J} / \mathrm{kg}^{3}$. It is easy to see in the figures that there are signatures of impulses of the first and second sounds at $t_{(1)} \approx r / U_{1}$ and $t_{(2)} \approx r / U_{2}$. The amplitudes of the impulses are large enough to be recorded by using modern methods.

\section{Harmonical law}

Let us now consider the case where the intensity of the laser beam changes in time with acoustical frequency $\omega$. It is instructive to carry out calculations using the complex functions

$$
\varphi(t)=\exp (-i \omega t), \quad \frac{\partial \varphi}{\partial t}=-i \omega \varphi(t),
$$

but keep in mind that we should finally take only the real parts. We assume that all the other variables vary according to the same law:

$$
\begin{aligned}
& \bar{P}^{\prime}(t, s)=\bar{P}^{\prime}(\omega, s) \exp (-i \omega t), \\
& \bar{T}^{\prime}(t, s)=\bar{T}^{\prime}(\omega, s) \exp (-i \omega t) .
\end{aligned}
$$

Using

$$
\frac{d^{2} \bar{P}^{\prime}}{d t^{2}}=-\omega^{2} \bar{P}^{\prime}(t, s), \quad \frac{d^{2} \bar{T}^{\prime}}{d t^{2}}=-\omega^{2} \bar{T}^{\prime}(t, s)
$$

in Eqs. (12) and (13), we obtain

$$
\begin{gathered}
{\left[\omega^{2}-C_{1}^{2} s^{2}\left(1+\frac{\rho_{s}}{\rho_{n}} \beta^{2}+\frac{\alpha_{T} \beta C_{2}^{2}}{\bar{\sigma}}\right)\right] \bar{P}^{\prime}(\omega, s)+} \\
+\frac{\rho_{0} \beta C_{P} C_{1}^{2} C_{2}^{2} s^{2}}{T_{0} \bar{\sigma}} \bar{T}^{\prime}(\omega, s)=\frac{\alpha I_{0} \beta C_{1}^{2} i \omega \varphi_{1}(s)}{T_{0} \sigma_{0}}, \quad(32) \\
+\frac{T_{0} s^{2}}{\rho_{0} C_{P}}\left[\alpha_{T}\left(C_{1}^{2}-C_{2}^{2}\right)-\frac{\rho_{s}}{\rho_{n}} \beta \bar{\sigma}+\frac{\rho_{s}}{\rho_{n}} \alpha_{T} \beta^{2} C_{1}^{2}+\right. \\
\left.\beta \alpha_{T}^{2} C_{1}^{2} C_{2}^{2}\right] \bar{P}^{\prime}(\omega, s)-\left[\omega^{2}-s^{2} C_{2}^{2}\left(1-\frac{\alpha_{T} \beta C_{1}^{2}}{\bar{\sigma}}\right)\right] \times \\
\times \bar{T}^{\prime}(\omega, s)=-\frac{i \omega\left(\bar{\sigma}-\beta \alpha_{T} C_{1}^{2}\right) \alpha I_{0} \varphi_{1}(s)}{\rho_{0} C_{P} \sigma_{0}} .
\end{gathered}
$$

The solution of Eq. (32) is similar to that of Eq. (16). It is given by

$$
\begin{gathered}
\bar{P}^{\prime}(\omega, s)= \\
=-\frac{i \alpha \omega I_{0} \beta C_{1}^{2} \varphi_{1}(s) \omega^{2}}{T_{0} \sigma_{0}\left[\omega^{4}-\omega^{2} s^{2}\left(C_{1}^{2}+C_{2}^{2}+\rho_{s} \rho_{n} \beta^{2} C_{1}^{2}\right)+C_{1}^{2} C_{2}^{2} s^{4}\right]}, \\
\bar{T}^{\prime}(\omega, s)= \\
=\frac{i \omega \alpha I_{0} \varphi_{1}(s)\left[\omega^{2}\left(\bar{\sigma}-\alpha_{T} \beta C_{1}^{2}\right)-C_{1}^{2} \bar{\sigma} s^{2}\right]}{\rho_{0} \sigma_{0} C_{P}\left[\omega^{4}-\omega^{2} s^{2}\left(C_{1}^{2}+C_{2}^{2}+\rho_{s} \rho_{n} \beta^{2} C_{1}^{2}\right)+C_{1}^{2} C_{2}^{2} s^{4}\right]} .
\end{gathered}
$$

The frequency $\omega$ must satisfy the equation

$$
\omega^{4}-\omega^{2} s^{2}\left(C_{1}^{2}+C_{2}^{2}+\frac{\rho_{s}}{\rho_{n}} \beta^{2} C_{1}^{2}\right)+C_{1}^{2} C_{2}^{2} s^{4}=0 .
$$

Its roots can be written as

$$
\omega_{1}^{2}=U_{1}^{2} s^{2}, \quad \omega_{2}^{2}=U_{2}^{2} s^{2} .
$$


Simple calculation allows one to express Eqs. (33) and (34) in the form

$\bar{P}^{\prime}(\omega, s)=\frac{i \omega \alpha I_{0} \beta U_{1}^{2} \varphi_{1}(s)}{T_{0} \sigma_{0}\left(U_{1}^{2}-U_{2}^{2}\right)}\left(\frac{1}{s^{2}-k_{1}^{2}}-\frac{1}{s^{2}-k_{2}^{2}}\right)$,

$\bar{T}^{\prime}(\omega, s)=-\frac{i \omega \alpha I_{0} \varphi_{1}(s) \bar{\sigma}}{\rho_{0} C_{P} \sigma_{0} U_{2}^{2}}\left(\frac{1+G_{2}}{s^{2}-k_{2}^{2}}-\frac{G_{1} U_{2}^{2}}{U_{1}^{2}} \frac{1}{s^{2}-k^{2}}\right)$.

The inverse Hankel transfer of Eqs. (37) and (38) yields

$$
\begin{aligned}
P^{\prime}(t, r)= & -\frac{\alpha \omega I_{0} \beta U_{1}^{2}}{4 T_{0} \sigma_{0}\left(U_{1}^{2}-U_{2}^{2}\right)}\left[H_{0}^{(1)}\left(k_{1} r\right) \exp \left(-\frac{k_{1}^{2} \omega^{2}}{8}\right)-\right. \\
& \left.-H_{0}^{(1)}\left(k_{2} r\right) \exp \left(-\frac{k_{2}^{2} w^{2}}{8}\right)\right] \exp (-i \omega t) \text {. (39) } \\
T^{\prime}(t, r)= & \frac{\alpha \omega I_{0} \bar{\sigma}}{4 \rho_{0} \sigma_{0} C_{P} U_{2}^{2}}\left[\left(1+G_{2}\right) H_{0}^{(1)}\left(k_{2} r\right) \exp \left(-\frac{k_{2}^{2} w^{2}}{8}\right)-\right. \\
- & \left.\frac{G U_{2}^{2}}{U_{1}^{2}} H_{0}^{(1)}\left(k_{1} r\right) \exp \left(-\frac{k_{1}^{2} w^{2}}{8}\right)\right] \exp (-i \omega t),
\end{aligned}
$$

where $H_{0}^{(1)}$ is the Hankel function. It is evident from Eqs. (39) and (40) that the system generates a cylindrical wave. In the far zone, where $r \gg U_{1,2} / \omega$, the needed expressions take the form

$$
\begin{gathered}
P^{\prime}(t, r)=-\frac{\alpha \omega I_{0} \beta U_{1}^{2} \exp (-i(\omega t+\pi / 4))}{2 T_{0} \sigma_{0}\left(U_{1}^{2}-U_{2}^{2}\right) \sqrt{2 \pi r}}\left[k_{1}^{-1 / 2} \times\right. \\
\left.\times \exp \left(i r k_{1}-\frac{k_{1}^{2} w^{2}}{8}\right)-k_{2}^{-1 / 2} \exp \left(i r k_{2}-\frac{k_{2}^{2} w^{2}}{8}\right)\right], \\
T^{\prime}(t, r)=\frac{\alpha \omega I_{0} \bar{\sigma} \exp (-i(\omega t+\pi / 4))}{2 \rho_{0} C_{P} \sigma_{0} U_{2}^{2} \sqrt{2 \pi r}}\left[\frac{1+G_{2}}{\sqrt{k_{2}}} \times\right. \\
\left.\times \exp \left(i r k_{2}-\frac{k_{2}^{2} w^{2}}{8}\right)-\frac{G_{1} U_{2}^{2}}{U_{1}^{2} \sqrt{k_{1}}} \exp \left(i r k_{1}-\frac{k_{1}^{2} w^{2}}{8}\right)\right] .
\end{gathered}
$$

The expressions obtained by us show that the amplitudes of excited oscillations are proportional to $\sqrt{\omega}$. There are maxima at the frequencies $\omega_{\max _{1,2}} \approx \sqrt{2} U_{1,2} / w$ in the dependence of the amplitudes of impulses on frequency. For $w=0.05 \mathrm{~cm}$ they are $\omega_{1} \approx 10^{6} \mathrm{~s}^{-1}$ and $\omega_{2} \approx 10^{5} \mathrm{~s}^{-1}$ for the first and second sound, respectively. Note that signals of heat impulses were reported [12] to be firmly recorded, and that their amplitude is of the order of $10^{-6} \mathrm{~K}$. This allows one to reduce $I_{0}$ by a factor of 10-100, while $w$ can be reduced to a tolerable possibility (with allowance for the diffraction effects). The maximal amplitudes of the recorded signal of the second sound can be increased up to $10^{7}-10^{8} \mathrm{~s}^{-1}$.

\section{Conclusions}

We have considered the possibility of an optical generation of the first and second sounds in the superfluid ${ }^{3} \mathrm{He}-{ }^{4} \mathrm{He}$ solutions. It has been shown that a synchronous generation of waves of the first and second sounds is quite realistic if a solution is illuminated by amplitude-modulated laser radiation with the wavelength corresponding to the range of absorption of the system. It has been shown that this method covers the range of frequencies of the second sound, which is hardly achievable by means of other methods.

I wish to thank Prof. V. P. Romanov for detailed discussions and Dr. A. A. Karabutov for useful advice.

1. I. M. Khalatnikov, Introduction to the Theory of Superfluids, Benjamin, New York (1965).

2. E. Ya. Rudavsky and I. A. Serbin, JETP 51, 1930 (1966).

3. E. M. Lifshitz, J. Phys. USSR 8, 110 (1944).

4. L. S. Dikina, E. Ya. Rudavsky, and I. A. Serbin, JETP 58, $843(1970)$

5. B. N. Yesel'son, M. I. Kaganov, E. Ya. Rudavsky, and I. A. Serbin, Usp. Fiz. Nauk 112, 591 (1974).

6. D. A. Rockwell, R. F. Benjamin, and T. J. Greytak, J. Low Temp. Phys. 18, 389 (1975).

7. L. M. Lyamshev, Laser Thermooptical Excitations of Sound, Nauka, Moscow (1989).

8. V. P. Romanov and T. Kh. Salikhov, Phys. Lett. A161, 161 (1991)

9. C. M. Surko, R. E. Packerd, G. J. Dick, and F. Reif, Phys. Rev. Lett. 24, 657 (1970).

10. M. Stockton, J. W. Keto, and W. A. Fitzsimmons, Phys. Rev. A5, 372 (1972)

11. B. N. Yesel'son, V. G. Ivantzov, V. A. Koval, E. Ya. Rudavsky, and I. A. Serbin, Properties of Liquid and Solid Helium. Solutions ${ }^{3} \mathrm{He}-{ }^{4} \mathrm{He}$, Kiev (1982).

12. N. E. Dyumin, N. N. Grigor'ev, and C. V. Svatko, Fiz. Nizk. Temp. 15, 253 (1989) [Sov. J. Low Temp. Phys. 15 142 (1989)] 